\title{
New 3-set Venn diagram
}

SIR-Recently, one of us (A.W.F. Edwards New Scient. 121, 51-56; 1989) gave a solution to the problem of constructing a Venn diagram for an arbitrary number of sets (Fig. 1a) and commented

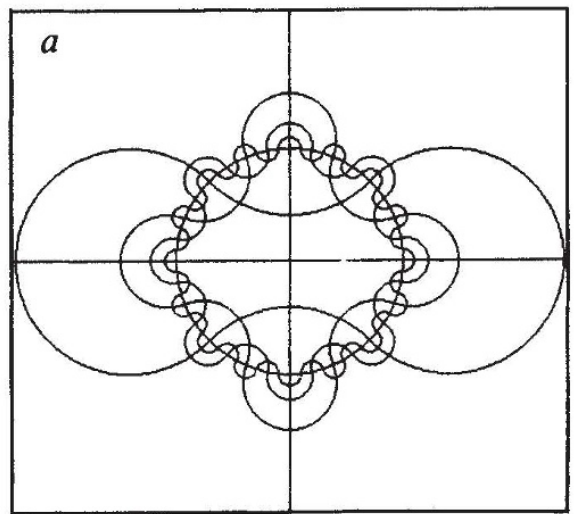

to be different to the usual form (Fig. 2).

It is easy to prove that there are no further forms for three sets, not counting as distinct any obtained by set boundaries merely touching without crossing. The

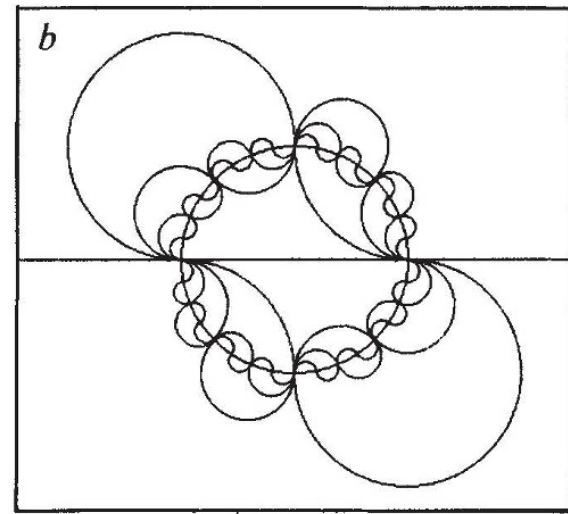

FIG. 1 a, The Edwards map of a 7-set Venn diagram. The boundaries of the first two sets are the straight lines; subsequent sets are concentric 'cogwheels' with $2,4,8, \ldots$ teeth, until the last set, which is the circle. $b$, An alternative version for six sets obtained by rotating the sets of the 7-set Edwards map. In the process the first two sets coincide, reducing the total number by one.
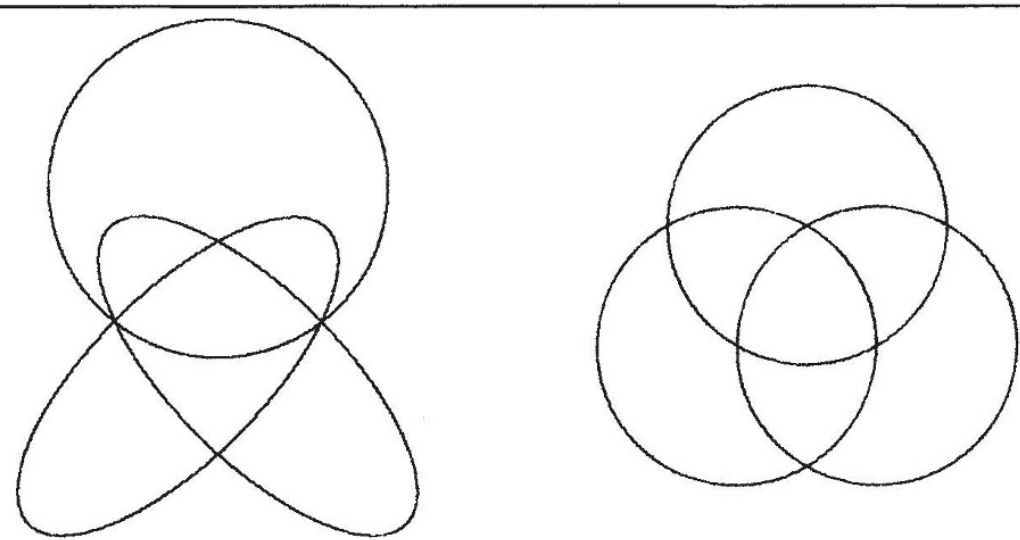

FIG. 2 The new form (left) of the Venn diagram for three sets compared with the usual form.

on the connection it revealed between Venn diagrams and the Gray code of communication theory. This prompted the other to remark that an equivalent representation of such an 'Edwards map' is the family of cosine curves $y=\left(\cos 2^{n} x\right) / 2^{n}, 0 \leqslant x \leqslant \pi, n=0,1,2, \ldots$, and that the family of sine curves $y=$ $\left(\sin 2^{n} x\right) / 2^{n}, 0 \leqslant x \leqslant 2 \pi, n=0,1,2, \ldots$, is an alternative which in turn reveals a similar connection with ordinary binary code. Although the association of these functions with the Gray and binary codes is not new, we believe their association with Venn diagrams is.

Because the sine curves are but phaseshifted cosine curves, the analogous version of the Edwards map is obtained by rotating each set of the map through an angle corresponding to one quarter of a wavelength (Fig. 1b). (A model made with sets copied onto transparent sheets and concentrically mounted is a useful aid.) When this is done, the topological form of the diagram for three sets is found number of forms for $k$ sets $(k>3)$ is still an open question.

\section{A.W.F. EDWARDS} C.A.B. SMITH

Gonville and Caius College,

Cambridge CB2 1TA, UK

\section{Pulsar structure}

SIR-The observed ${ }^{1,2}$ properties of PSR0535-69 (the SN1987A pulsar) may be explained if the neutron star is internally convecting or stirred. I reject the apparently natural interpretation of the 8hour period as an orbital period because the projected semimajor axis would be smaller than the progenitor star; it is not possible for a Jupiter-like companion (implied by the small mass function) to survive deep inside another star, and matter from a stellar interior has too much entropy to condense into such an object.

I suggest that the roughly sinusoidal variations may be timing noise resembling that observed (at lower amplitude) in many older pulsars. This hypothesis naturally explains the discrepancies between the pure sinusoid and the data, and predicts that the period variations will not remain sinusoidal.

Alternatively, the sinusoidal part of the period variations may be attributed to torsional magnetohydrodynamic oscillations of the neutron star's crust with respect to its interior, or to Tkhachenko modes ${ }^{3}$. Magnetohydrodynamic oscillations would be expected to have periods of the order of $r\left(8 \pi \varrho_{\text {eff }} / B^{2}\right)^{1 / 2}$, where $r$ is the radius, $\varrho_{\text {eff }}$ is the density and $B$ is the magnetic field of the neutron star. Adopting $\varrho_{\text {eff }} \approx 10^{14} \mathrm{~g} \mathrm{~cm}^{-3}$ and $r=10 \mathrm{~km}$ yields $B \approx$ $2 \times 10^{9}$ gauss. Even if oscillations are present the non-sinusoidal part of the residuals implies the existence of timing noise.

Independent estimates of the magnetic field are consistent with the low values required by the torsional oscillation model. First, the reported bound on the period derivative implies an effective magnetic moment $\mu<10^{29} I_{45}^{1 / 2} \mathrm{G} \mathrm{cm}^{3}$, where $I_{45}$ is the moment of inertia of the neutron star in units of $10^{45} \mathrm{~g} \mathrm{~cm}^{2}$. Second, the optical light curve of SN1987A suggests $^{4}$ an estimate of $\sim 10^{38} \mathrm{erg} \mathrm{s}$ for the pulsar contribution, implying $\mu \approx 4 \times 10^{26}$ $\varepsilon^{-1 / 2} \mathrm{G} \mathrm{cm}^{3}$, where $\varepsilon$ is the efficiency of converting spin-down power to visible radiation. Unless $\varepsilon$ is extremely small $\left(<10^{-7}\right), \mu$ is less than typical pulsar values. I conclude that PSR0535-69 was born spinning rapidly and with a low magnetic field, thus obviating the need ${ }^{5}$ for accretional spin up in a binary system to produce such an object.

If the surface layers of the neutron star are undergoing convection then both the pulse intensity variations (and disappearance) and the timing noise may be explained. Magnetic flux is frozen on the required timescale (hours), so subduction and upwelling of surface material will change the exterior field configuration, and hence will alter the pulse power and angular distribution. Surface convection will also produce fluctuations in $I$, explaining the observed timing noise. If the surface is solid ${ }^{3.6}$, strains will be imposed and relax and 'mountains' will rise and fall, in a similar manner to terrestrial plate tectonics. Even liquid matter will not be exactly barytropic because hysteresis in achieving $\beta$-equilibrium (a source of substantial bulk viscosity) means that convection could also lead to fluctuations in $I$. A number of forces may drive convection or stir the neutron star - cooling of upper layers by neutrino bremsstrahlung ${ }^{6}$, Eddington-Sweet currents resulting from variation of cooling rates with latitude (pronounced in such a rapidly rotating object), possible internal differential rotation and so on.

Fluctuations in $I$ of magnitude $\sim 10^{-6} I$ are inferred from the spin rate. If the 